\title{
Mobile parallel robot machine for heavy-duty double-walled vacuum vessel assembly
}

\author{
Changyang Li and Huapeng Wu \\ Laboratory of Intelligent Machines, LUT University, Lappeenranta, Finland \\ Harri Eskelinen \\ Laboratory of Production Engineering, LUT University, Lappeenranta, Finland, and \\ Haibiao fi \\ College of Physics and Optoelectronic Engineering, Shenzhen University, Shenzhen, China
}

\begin{abstract}
Purpose - This paper aims to present a detailed mechanical design of a seven-degrees-of-freedom mobile parallel robot for the tungsten inert gas (TIG) welding and machining processes in fusion reactor. Detailed mechanical design of the robot is presented and both the kinematic and dynamic behaviors are studied.

Design/methodology/approach - First, the model of the mobile parallel robot was created in computer-aided design (CAD) software, then the simulation and optimization of the robot were completed to meet the design requirements. Then the robot was manufactured and assembled. Finally, the machining and tungsten inert gas (TIG) welding tests were performed for validation.

Findings - Currently, the implementation of the robot system has been successfully carried out in the laboratory. The excellent performance has indicated that the robot's mechanical and software designs are suitable for the given tasks. The quality and accuracy of welding and machining has reached the requirements.

Originality/value - This mobile parallel industrial robot is particularly used in fusion reactor. Furthermore, the structure of the mobile parallel robot can be optimized for different applications.
\end{abstract}

Keywords Parallel manipulator, Welding, Machining

Paper type Case study

\section{Introduction}

The China Fusion Engineering Test Reactor (CFETR) is a tokamak nuclear fusion reactor in China. The CFETR is proposed to be built and operated during the gap between the International Thermonuclear Experimental Reactor (ITER) and the Demonstration Power Station (DEMO). To meet the demands on effective and safe use of fusion reactor, the remote handling technology is inevitably required in assembling and maintenance. A design of robot for the maintenance of divertor in the DEMO fusion reactor and a design of mobile parallel robot for assembling and machining the fusion reactor's vacuum vessel (VV) are discussed by the same research group in Li et al. $(2019,2020)$.

The CFETR VV shown in Figure 1 (left) consists of eight 1/8 sectors. Each sector is welded and assembled from two 1/16 sectors with a transitional segment. The transitional segment shown in Figure 1 (right) consists of two splice plates:

1 one splice plate in the outer shell is $50-\mathrm{mm}$ thick and 100mm wide; and

The current issue and full text archive of this journal is available on Emerald Insight at: https://www.emerald.com/insight/0143-991X.htm

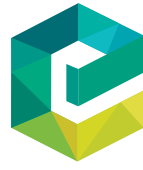

Industrial Robot: the international journal of robotics research and application 48/4 (2021) 523-531

Emerald Publishing Limited [ISSN 0143-991X]

[DOI 10.1108/IR-10-2020-0228]
2 one splice plate in the inner shell is $50-\mathrm{mm}$ thick and 200-mm wide.

The distance between the inner and outer shell is unequal because of the different neutron irradiation on the double-wall structure (Song et al., 2014).

The task of the mobile parallel robot is to perform the machining and (tungsten inert gas) TIG welding along an exact path during the assembly operation. Because of the machining force and the tools' weights, a normal series robot cannot offer sufficient stiffness with the required accuracy. The parallel robot has relatively higher stiffness and load capability than series robot (Stewart, 1965; Wang et al., 2001). Moreover, a carriage is

(C) Changyang Li, Huapeng Wu, Harri Eskelinen and Haibiao Ji. Published by Emerald Publishing Limited. This article is published under the Creative Commons Attribution (CC BY 4.0) licence. Anyone may reproduce, distribute, translate and create derivative works of this article (for both commercial \& non-commercial purposes), subject to full attribution to the original publication and authors. The full terms of this licence may be seen at http://creativecommons.org/licences/by/4.0/ legalcode

This work has been carried out within the framework of the EUROfusion Consortium and has received funding from the Euratom Research and Training Programme 2014-2018 and 2019-2020 under the grant agreement no. 633053. The views and opinions expressed herein do not necessarily reflect those of the European Commission.

Received 20 October 2020

Revised 11 January 2021

3 February 2021

Accepted 4 February 2021 
Figure 1 Three-dimensional schematic of VV sectors assembly (left) and splice spates locations (right)

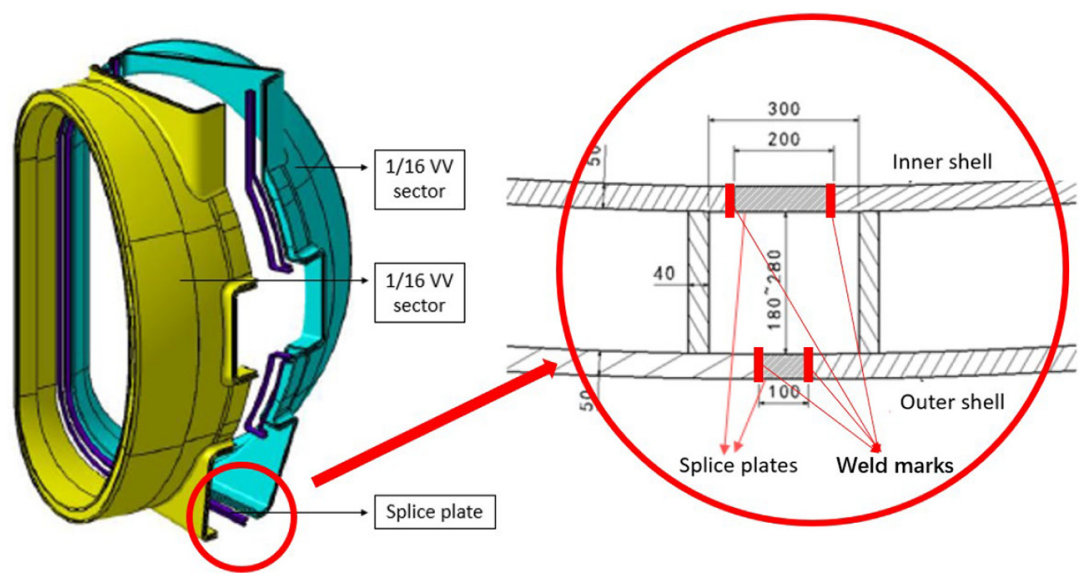

integrated into the robot system to provide linear motion along the track rail for improving mobility. The robot system was designed and built, the welding and machining tests were performed in the lab and the results of the tests have met the requirements. Being compared with the similar ten-DoF mobile parallel robot in Figure 2 , developed by the same research group and demonstrated in ITER (Pessi et al., 2007; Wu et al., 2008), the newly designed robot machine is more reliable. This newly designed robot is a seven-DoF robot, and it has relatively less components but can perform the required tasks. Its simple structure makes the maintenance of the robot easier and saves space for installing other components in the VV, such as the track rail.

\section{Kinematic model of robot}

The mobile parallel robot shown in Figure 3 consists of two sub-structures, namely, the carriage and the parallel

Figure 2 Ten-DoF parallel robot

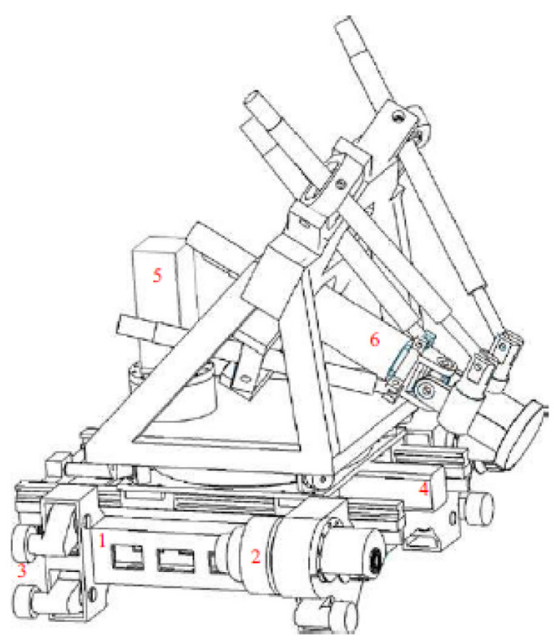

Notes: (1) Carriage frame; (2) tracking drive unit; (3) compensation mechanism; (4) linear drive unit; (5) rotation drive unit; (6) Penta-WH parallel mechanism Source: Wu et al. (2008) manipulator. The welding process is carried out continuously along the track rail mounted on the inner surface of the VV sector to ensure continuous weld joint. The machining process is carried out in cycles and the robot can provide high stiffness and high machining accuracy.

The required workspace is $200 \times 200 \times 300 \mathrm{~mm}(x-y-z)$, but the real workspace of the robot is bigger than this requirement. The robot system carries out machining, welding and the nondestructive testing processes when different tools are mounted on the movable platform. The dynamic force comes from the milling process is up to $500 \mathrm{~N}$ and in transportation process the robot needs to carry the splice plate, whose weight is $150 \mathrm{~kg}$. Thus, in the designing consideration, a force of $2,000 \mathrm{~N}$ is applied to the movable platform in the $x, y$ and $z$ directions, and this produces resultant force of $3.5 \mathrm{kN}$. Also, the robot should reach the requirements of working with $0.1-\mathrm{mm}$ movement and milling accuracy.

\subsection{Carriage}

The carriage moves along the track rail mounted on the VV to any position inside the VV by means of rack and pinions from the lower port. The curvatures of track rail under the front and rear wheels of the carriage are different when the carriage enters the curve path, so two motors control the front and rear wheels independently. The carriage has adopted the non-clearance double-gear output transmission mechanism (Pessi et al., 2007). The clearance compensation mechanism and the fastening wheels contribute to the smooth movement for

Figure 3 Mobile parallel robot

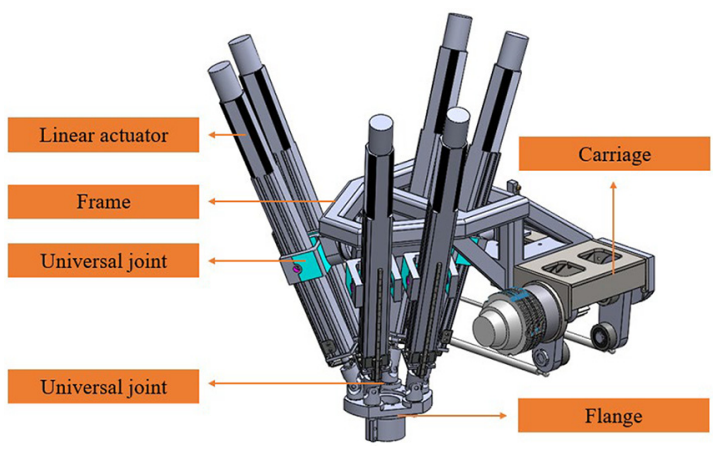


avoiding vibration when the carriage moves. The positioning of the carriage is decided by an independent rack mounted in the middle of the track rail. The encoder is integrated into the carriage. The simulation of the robot motion path on the track rail is shown in Figure 4 (left). Once the robot reaches the designated position, the parallel manipulator starts its task. The limited space can be seen in Figure 4 (right). The carriage is mounted on the track rail and is close to the side surface of the VV. When there are cables connected, the space will be even narrower. To avoid collision in the structure and components, we leave clearance so that the parallel manipulator has enough workspace.

The main body of carriage is composed of several welded steel plates, so that it has enough stiffness to stand the payload within the limited deflection and offer enough space for maintaining the mechanism. One side of the carriage is connected to the parallel manipulator by bolting and the other side of the carriage has space for wheel units. Other machine, such as series robot, can be mounted on the carriage if task requires. The encoder part is mounted in the middle of the carriage; while the beltpulley structure is adopted to enable the encoder gear reach the rack in such a limited space. The gas spring allows the encoder gear to move in the curved track rail. In the clearance compensation system, i.e. the spring shown in Figure 5, the extension gas spring pushes the two-wheeled unit to both ends. In this way, the wheels are always clamped on to the track rail during the movement, even in the vertical position.

The track rail shown in Figure 6 is a guide rail mounted on the inner surface of the VV so that the robot can move inside the VV. There are four sub-rails on the track rail: the two subrails at both sides are $\mathrm{V}$-shaped rail and flat rail, the thinner rack in the middle is for encoder gear, and the thicker rack in the middle is for the main motion driven gear, i.e. the transmission gear. This configuration eliminates the robot movement in the horizontal direction thanks to the $\mathrm{V}$-shaped rail.

\subsection{Parallel manipulator}

The parallel manipulator in Figure 3 is designed based on the Stewart platform. The rotations and translations along the $x, y$ and $z$ directions contribute to six DOFs. It is bolted with the carriage, while the movable platform is linked to the base platform by six servo electrical actuators. There are six universal joints on the base and movable platforms, respectively, providing the flexibility for the robot. Different tools, such as spindle, the welding equipment and the

Figure 4 Robot movement simulation on the track rail
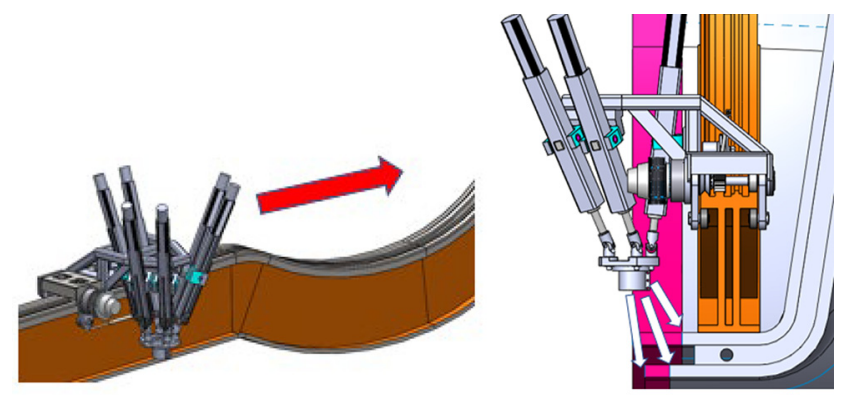

Notes: (1) Carriage (left); (2) parallel manipulator (right) visualization equipment, can be installed in the flange to perform different tasks.

\section{Kinematic model}

In the kinematic model, both the carriage and the parallel manipulator are sub-assemblies. The carriage provides one degrees of freedom (DoF) along the track rail and the parallel manipulator contributes six degrees of freedom DoFs. The kinematic model of the parallel manipulator is shown in Figure 7.

According to the geometry of the platforms in Figure 7, the distance vector of each limb and the rotation matrix are obtained as in equations (1) and (2):

$$
\begin{gathered}
\vec{L}_{i}=\vec{r}_{a_{i}, P}+\vec{r}_{P O}+\vec{r}_{O, b_{i}}(i=1,2,3,4,5,6) \\
R=\left[\begin{array}{ccc}
c \alpha c \beta & c \alpha s \beta s \gamma-s \alpha c \gamma & c \alpha s \beta c \gamma+s \alpha s \gamma \\
s \alpha c \beta & s \alpha s \beta s \gamma+c \alpha c \gamma & s \alpha s \beta c \gamma-c \alpha s \gamma \\
-s \beta & c \beta s \gamma & c \beta c \gamma
\end{array}\right]
\end{gathered}
$$

Where $R$ is the rotation matrix with respect to frame $P-X_{2} Y_{2} Z_{2}$; $\alpha, \beta$ and $\gamma$ are the yaw, pitch and roll angles around the $x, y$ and $z$ axis in frame $P-X_{2} Y_{2} Z_{2} ; \vec{r}_{a_{i}, P}$ is the distance vector of joint of the $i$ th limb on the mobile platform with respect to frame $P-X_{2} Y_{2} Z_{2} ; \vec{r}_{O, b_{i}}$ is the vector of joint of the $i$ th cylinder with respect to frame $O-X_{1} Y_{1} Z_{1}$; and $\vec{r} P O$ is the distance vector

\section{Figure 5 Carriage}

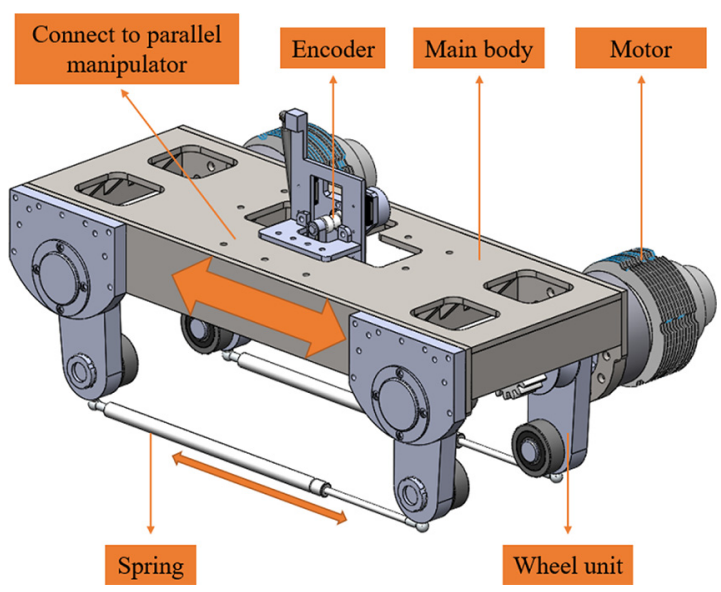

Figure 6 Track rail

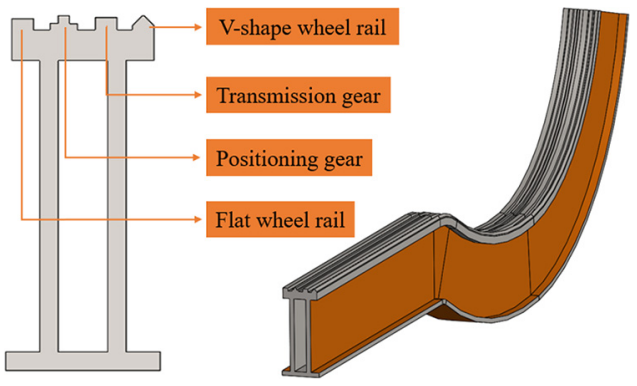


Figure 7 Kinematic model of the parallel manipulator

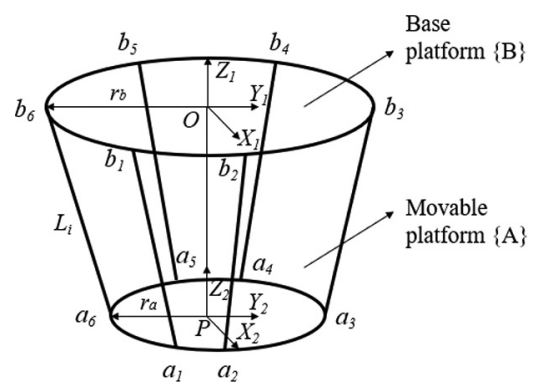

in global frame. The length of each limb defined with respect to frame $P-X_{2} Y_{2} Z_{2}$ is in determined in equation (2):

$$
l_{i}=\sqrt{\left(R_{a_{i}, P}+\vec{r}_{P O}+\vec{r}_{O, b_{i}}\right)^{T}\left(R_{a_{i}, P}+\vec{r}_{P O}+\vec{r}_{O, b_{i}}\right)}
$$

With the above equations and given geometry information, it is possible to calculate: the platform location and orientation; and the stroke of each actuator.

\section{Dynamic model}

The dynamic analysis focuses on the parallel manipulator. It calculates the actuator force to choose suitable actuator. Moreover, by tuning the parameters, such as the geometries of base and movable platform, the multi-objective optimization of the parallel manipulator can be carried out and a new structure can be designed for specific task. The closed-form dynamic formulation is adopted in our dynamic analysis (Ting et al., 2004; Merlet, 2006). The actuator force is calculated by using equation (4):

$$
\tau=\mathcal{F}^{-T} *(M(\chi) * \chi+C(\chi) * \chi+G(\chi))
$$

Where:

$\tau$ : actuator force;

f: Jacobian matrix;

$M$ : mass matrix;

$C$ : Coriolis and centrifugal matrix; and

$G$ : gravity vector.

$\chi$ denotes a set of generalized coordinates for the position and orientation of point $P$ in the movable platform. The position, velocity and acceleration of point $P$ in $x, y$ and $z$ directions are denoted in a $3 \times 1$ matrix as $x_{p}, v_{p}$ and $a_{p}$, while the angular position, angular velocity and angular acceleration of point $P$ in $x, y$ and $z$ directions are denoted in a $3 \times 1$ matrix as $\theta, \omega, \omega$. Therefore, equations (5)-(7) are time derivative representations of $\chi$.

$$
\begin{aligned}
& \chi=\left[\begin{array}{c}
x_{p} \\
\theta
\end{array}\right] \\
& \chi=\left[\begin{array}{c}
v_{p} \\
\omega
\end{array}\right]
\end{aligned}
$$

$$
\chi=\left[\begin{array}{l}
a_{p} \\
\omega
\end{array}\right]
$$

\subsection{Jacobian matrix}

The Jacobian matrix reveals the relation between the joints of the parallel manipulator to the movable platform and constructs the transformation to calculate the actuator forces from the forces and moments applied on the movable platform. Two coordinate frames $\{A\}$ and $\{B\}$ are movable and base platform, respectively, shown in Figure 7, and their origins are located at centroids $\{P, O\}$ of the platforms; the vectors ${ }^{A} a$ and ${ }^{B} b$ describe the position of the reference points locations, which is shown in equation (8):

$$
A_{a}=\left[\begin{array}{lll}
a_{1 x} & a_{1 y} & a_{1 z} \\
a_{2 x} & a_{2 y} & a_{2 z} \\
a_{3 x} & a_{3 y} & a_{3 z} \\
a_{4 x} & a_{4 y} & a_{4 z} \\
a_{5 x} & a_{5 y} & a_{5 z} \\
a_{6 x} & a_{6 y} & a_{6 z}
\end{array}\right]{ }^{B} b=\left[\begin{array}{lll}
b_{1 x} & b_{1 y} & b_{1 z} \\
b_{2 x} & b_{2 y} & b_{2 z} \\
b_{3 x} & b_{3 y} & b_{3 z} \\
b_{4 x} & b_{4 y} & b_{4 z} \\
b_{5 x} & b_{5 y} & b_{5 z} \\
b_{6 x} & b_{6 y} & b_{6 z}
\end{array}\right]
$$

The position of the movable platform in relation to time $t$ is denoted in equation (9), where variables $x_{0}, y_{0}, z_{0}$ are the initial positions of $P$ relative to the point $O$, and $\Delta x(t), \Delta y(t)$ and $\Delta z(t)$ are the displacements of point $P$ relative to point $O$ in time $t$.

$$
P(t)=\left[\begin{array}{c}
x_{0}+\Delta x(t) \\
y_{0}+\Delta y(t) \\
z_{0}+\Delta z(t)
\end{array}\right]
$$

The unit vector in the direction of limb $i$ is calculated in equation (10), where $R$ refers to the rotation matrix in equation (2), while $\alpha, \beta$ and $\gamma$ are variables that change with time $t$.

$$
\hat{s}_{i}=\frac{P(t)+R *{ }^{A} a_{i}-{ }^{B} b_{i}}{l_{i}}
$$

Then Jacobian matrix can be denoted as equation (11):

$$
\mathcal{J}=\left[\begin{array}{ll}
\hat{s}_{1}^{T} & \left(b_{1} \times \hat{s}_{1}\right)^{T} \\
\hat{s}_{2}^{T} & \left(b_{2} \times \hat{s}_{2}\right)^{T} \\
\hat{s}_{3}^{T} & \left(b_{3} \times \hat{s}_{3}\right)^{T} \\
\hat{s}_{4}^{T} & \left(b_{4} \times \hat{s}_{4}\right)^{T} \\
\hat{s}_{5}^{T} & \left(b_{5} \times \hat{s}_{5}\right)^{T} \\
\hat{s}_{6}^{T} & \left(b_{6} \times \hat{s}_{6}\right)^{T}
\end{array}\right]
$$

Where:

$$
b=R *^{B} b+P(t)
$$

After Jacobian matrix is calculated, several symbols are used to represent the physical parameters of the parallel robot; related terms of limbs can also be seen in Figure 8.

$m$ : mass of the movable platform;

$m_{i 1}$ : mass of cylinder;

$m_{i 2}:$ mass of piston; 
$c_{i 1}$ : half-length of cylinder; and

$c_{i 2}$ : half-length of piston.

Matrix transformations are denoted as equation (13):

$$
{ }^{A} I=R *^{B} I * R^{T}
$$

Where:

$$
{ }^{B} I=\left[\begin{array}{ccc}
I_{x x} & 0 & 0 \\
0 & I_{y y} & 0 \\
0 & 0 & I_{z z}
\end{array}\right]
$$

Kinematic analysis of each joint and limb are presented below. First, the linear velocity of point $P$ is denoted in equation (15), then the velocity and linear acceleration of point $b_{i}$ is denoted in equations (16) and (17). The angular velocity and acceleration of limb is denoted in equations (18) and (19). The linear velocity and acceleration of the mass center of each limb are denoted in equations (20)-(23).

$$
\begin{gathered}
v_{p}=\dot{P} \\
v_{b i}=v_{p}+\omega \times b_{i} \\
a_{b i}=a_{p}+\dot{\omega} \times b_{i}+\omega \times\left(\omega \times b_{i}\right) \\
\omega_{i}=\frac{\hat{s}_{i} \times v_{b i}}{L_{i}} \\
\dot{\omega}_{i}=\frac{\hat{s}_{i} \times a_{b i}-2 * l_{i} * \omega_{i}}{l_{i}} \\
v_{i 1}=c_{i 1} *\left(\omega_{i} \times \hat{s}_{i}\right) \\
v_{i 2}=\left(l_{i}-c_{i 2}\right) *\left(\omega_{i} \times \hat{s}_{i}\right)+l_{i} * \hat{s}_{i}
\end{gathered}
$$

Figure 8 Free-body diagram of the limbs

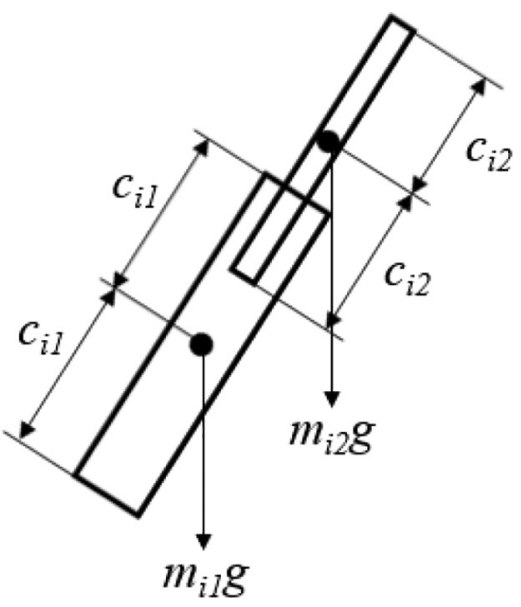

$$
a_{i 1}=c_{i 1} *\left(\omega_{i} \times \hat{s}_{i}+\omega_{i} \times\left(\omega_{i} \times \hat{s}_{i}\right)\right)
$$

$$
a_{i 2}=\left(l_{i}-c_{i 2}\right) *\left(\omega_{i} \times \hat{s}_{i}-\left(\omega_{i} \cdot \omega_{i}\right) * \hat{s}_{i}\right)+2 * l_{i} *\left(\omega_{i} \times \hat{s}_{i}\right)+l_{i} * \hat{s}_{i}
$$

\subsection{Mass matrix}

The mass matrix is defined from the kinetic energy of system and derived by substituting equations (25)-(28) into equation (24):

$$
\begin{gathered}
M(\chi)=M_{p}+\sum_{i=1}^{i=6} M_{l i} \\
M_{p}=\left[\begin{array}{cc}
m * I_{3 \times 3} & 0_{3 \times 3} \\
0_{3 \times 3} & A_{I}
\end{array}\right]_{6 \times 6} \\
M_{l i}=\mathcal{F}_{i}^{T} * M_{i} * \mathcal{F}_{i} \\
M_{i}=m_{i 2} * \hat{s}_{i} * \hat{s}_{i}^{T}-\frac{I_{x x i} * \hat{s}_{i \times}^{2}}{l_{i}^{2}}-m_{c e} * \hat{s}_{i \times}^{2} \\
m_{c e}=\frac{m_{i 1} * c_{i 1}^{2}+m_{i 2} * c_{i 2}^{2}}{l_{i}^{2}}
\end{gathered}
$$

Where:

$M_{p}$ : mass matrix of movable platform; and

$M_{l i}$ : mass matrix of limbs $i(i=1,2,3,4,5,6)$.

\subsection{Coriolis and centrifugal matrix}

The Coriolis and centrifugal matrix includes all the inertial forces caused by the Coriolis and centrifugal accelerations, and the mass matrix components are differentiated with respect to the motion variable $\chi$. Therefore, the Coriolis and centrifugal matrix is derived by substituting equations (30)-(33) into equation (29):

$$
C(\chi)=C_{p}+\sum_{i=1}^{i=6} C_{l i}
$$

$$
\begin{gathered}
C_{p}=\left[\begin{array}{cc}
0_{3 \times 3} & 0_{3 \times 3} \\
0_{3 \times 3} & \omega \times{ }^{A} I
\end{array}\right]_{6 \times 6} \\
C_{l i}=\mathcal{F}_{i}^{T} * M_{i} * \mathcal{F}_{i}+\mathcal{F}_{i}^{T} * C_{i} * \mathcal{F}_{i} \\
C_{i}=-\frac{2 * m_{c o} * l_{i} * \hat{s}_{i \times}^{2}}{l_{i}}-\frac{m_{i 2} * c_{i 2} * \hat{s}_{i} * x_{i}^{T} * \hat{s}_{i \times}^{2}}{l_{i}} \\
m_{c o}=\frac{m_{i 2} * c_{i 2}}{l_{i}}-\frac{I_{x x}+l_{i}^{2} * m_{c e}}{l_{i}^{2}}
\end{gathered}
$$

Where:

$C_{p}$ : mass matrix of movable platform; and

$C_{l i}$ : mass matrix of limbs $i(i=1,2,3,4,5,6)$. 


\subsection{Gravity vector}

The gravity vector is derived by substituting equations (35)-(38) into equation (34):

$$
\begin{gathered}
G(\chi)=G_{p}+\sum_{i=1}^{i=6} G_{l i} \\
G_{p}=\left[\begin{array}{c}
-m * g \\
0_{3 \times 3}
\end{array}\right]_{6 \times 1} \\
G_{l i}=\mathcal{F}_{i}^{T} * G_{i} \\
G_{i}=\left(m_{g e} * \hat{s}_{i \times}^{2}-m_{i 2} * \hat{s}_{i} * \hat{s}_{i}^{T}\right) * g \\
m_{g e}=\frac{\left.m_{i 1} * c_{i 1}+m_{i 2} *\left(l_{i}-c_{i 2}\right)\right)}{l_{i}}
\end{gathered}
$$

Where:

$G_{p}$ : mass matrix of movable platform; and
Volume $48 \cdot$ Number $4 \cdot 2021 \cdot 523-531$

$G_{l i}$ : mass matrix of limbs $i(i=1,2,3,4,5,6)$.

\section{Dynamic and finite element analysis}

The dynamic analysis is carried out in MATLAB software, and the finite element analysis is carried out to verify the structure's total deformation and equivalent stress.

\subsection{Dynamic analysis}

Dynamic model of the mobile parallel robot is built in MATLAB Simulink as shown in Figure 9. The coordinates of points $a_{i}(i=1,2,3,4,5,6)$ and $b_{i}(i=1,2,3,4,5,6)$ refer to point $O$ in Figure 7 are shown in Table 1 . The mass properties of each component and load are shown in Table 2.

The inputs are the rotation $\left(R_{x}, R_{y}, R_{z}\right)$ and linear $\left(D_{x}, D_{y}, D_{z}\right)$ movements of the movable platform in $x, y$ and $z$ directions, as expressed in equations (39) and (40). The coefficient 0.1 is the amplitude of the rotation angle and displacement of the movable platform and constant 3 is related to the period of the sine wave. The parameters chosen are for illustration convenience.

Figure 9 Dynamic simulation with PID control

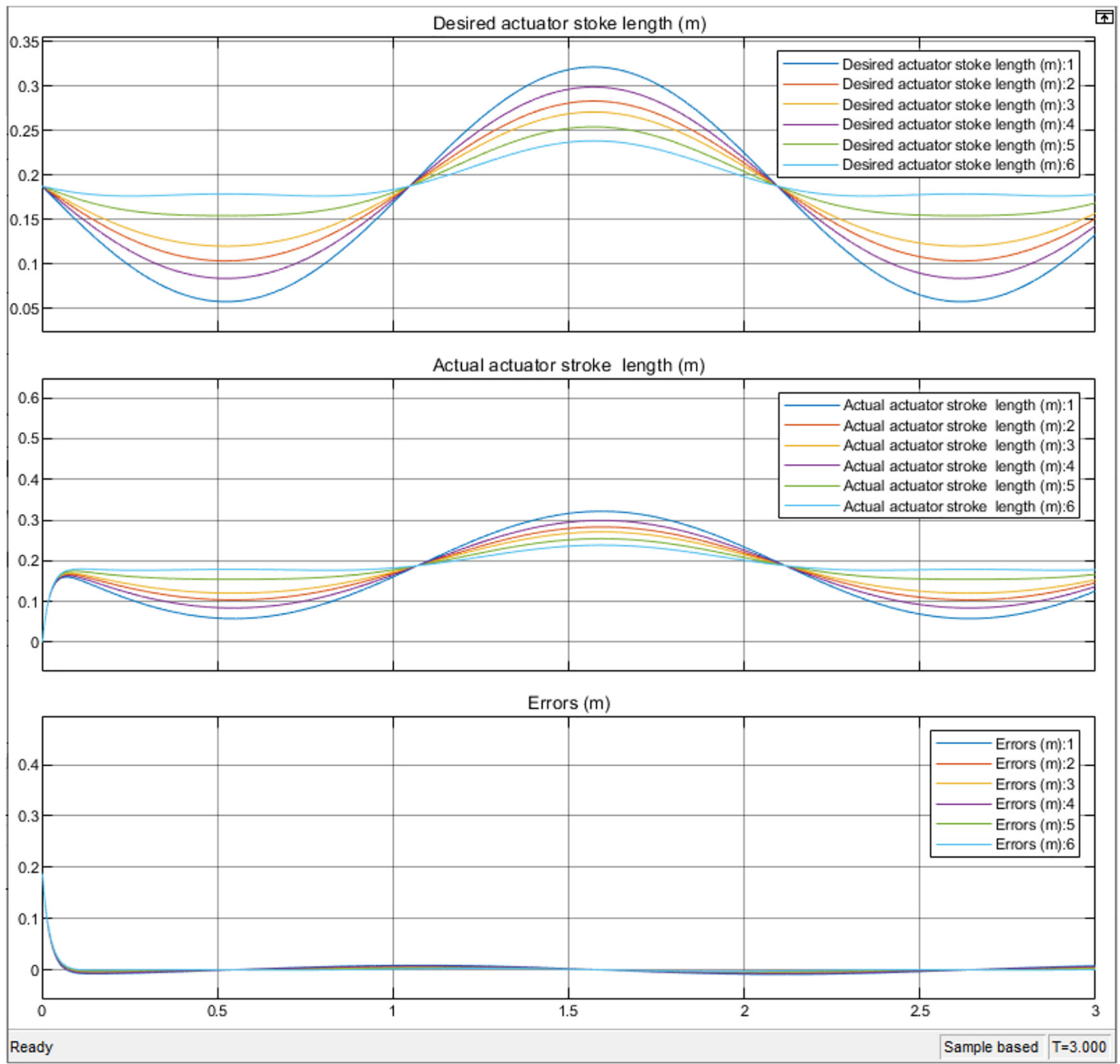




$$
\begin{gathered}
R_{x}=-0.1 * \sin (3 * t) \\
R_{y}=R_{z}=D_{x}=D_{y}=D_{z}=0.1 * \sin (3 * t)
\end{gathered}
$$

In the original position, the movable platform with $200 \mathrm{~kg}$ load is $0.4-\mathrm{m}$ below the base platform without any rotation in equilibrium position. After setting the movement of the movable platform, Figure 9 shows the length of each of the six actuators changes during the period of $3 \mathrm{~s}$. The desired actuator length is shown on the top of the figure, while the actual actuator length with the proportional-integral-derivative (PID) control is shown in the middle of the figure. The proportionalintegral-derivative (PID) parameters were selected to meet the required overshot $(\leq 5 \%)$ and the static response error $(\leq 2 \%)$. The differences between the desired and actual actuator stroke lengths are shown at the bottom of Figure 9.

\subsection{Finite element analysis}

The finite element analysis was carried out with the ANSYS workbench software. The idea was to verify the structure's total deformation and equivalent stress when the milling force is applied to the movable platform. Because of the smooth mesh consideration, the model is simplified without the loss of accuracy, for example, bolts are modeled using the software internal feature without any real geometry modelling, and the fillets of the structure benefits to the assembly are removed in some locations. The welded components are bonded. The fixed supports are set on the locations of bearings' and motors' shown in Figure 10 after
Industrial Robot: the international journal of robotics research and application Volume $48 \cdot$ Number $4 \cdot 2021 \cdot 523-531$

considering the force transmission in the wheels. A resultant force of $2,000 \mathrm{~N}$ in the $x, y$ and $z$ directions is applied in the frame of parallel robot. The material is $\mathrm{S} 355$ and the gravity force is applied (Li et al., 2020). The results of total deformation and equivalent stresses are shown in Figure 10. The highest equivalent stress is 18.088 MPa, and this is acceptable for the S355 material. The maximum of total deformation is $0.0515 \mathrm{~mm}$, and it occurs at the edge of the parallel robot frame.

\section{Experiment}

In this experiment, the control system consists of the motion control system and the task-based control system. The hardware is based on the industrial PC (IPC) control system. The EtherCAT with standard protocol connects all the I/O drivers, which is beneficial to editing and adding new functions. In addition, other hardware systems (milling, narrow gap welding and the non-singular terminal sliding mode (NTSM)) and computer (simulator, computer-aided design (CAD)/ computer-aided manufacturing (CAM) system) can be linked online (Feng et al., 2002; Li et al., 2013).

The carriage and the parallel robot can be controlled by either software or handwheel to ensure the system's reliability. Figure 11 shows the hardware of the control system and Figure 12 shows the control system, the milling process simulation and the real robot machine.

In the experiments, the tungsten inert gas (TIG) welding and machining are verified so that the results meet the requirements. It is observed that the carriage can move along

Table 1 Coordinates of base platform and movable platform at initial position ( $x, y$ and $z$ coordinates)

\begin{tabular}{lrrrrrr}
\hline Point & Point 1 $(\mathrm{mm})$ & Point 2 $(\mathrm{mm})$ & \multicolumn{1}{c}{ Point 3 $(\mathrm{mm})$} & Point 4 $(\mathrm{mm})$ & \multicolumn{1}{c}{ Point 5 $(\mathrm{mm})$} & \multicolumn{1}{c}{ Point 6 (mm) } \\
\hline $\boldsymbol{b}_{\boldsymbol{i}}$ & $(-151,-185,0)$ & $(151,-185,0)$ & $(236,-38,0)$ & $(85,233,0)$ & $(-85,233,0)$ & $(-236,-38,0)$ \\
$a_{i}$ & $(-34,-93,-500)$ & $(34,-93,-500)$ & $(-64,76,-500)$ & $(64,76,-500)$ & $(98,17,-500)$ & $(-98,17,-500)$ \\
\hline
\end{tabular}

Table 2 Mass properties of components

\begin{tabular}{lcccc}
\hline Parameter & Base platform $(\mathrm{kg})$ & Movable platform $(\mathrm{kg})$ & External load $(\mathrm{kg})$ & Actuator $(\mathrm{kg} / \mathrm{per})$ \\
\hline Mass & 100 & 10 & 200 & 10 \\
\hline
\end{tabular}

Figure $10 \mathrm{FE}$ analysis of structure
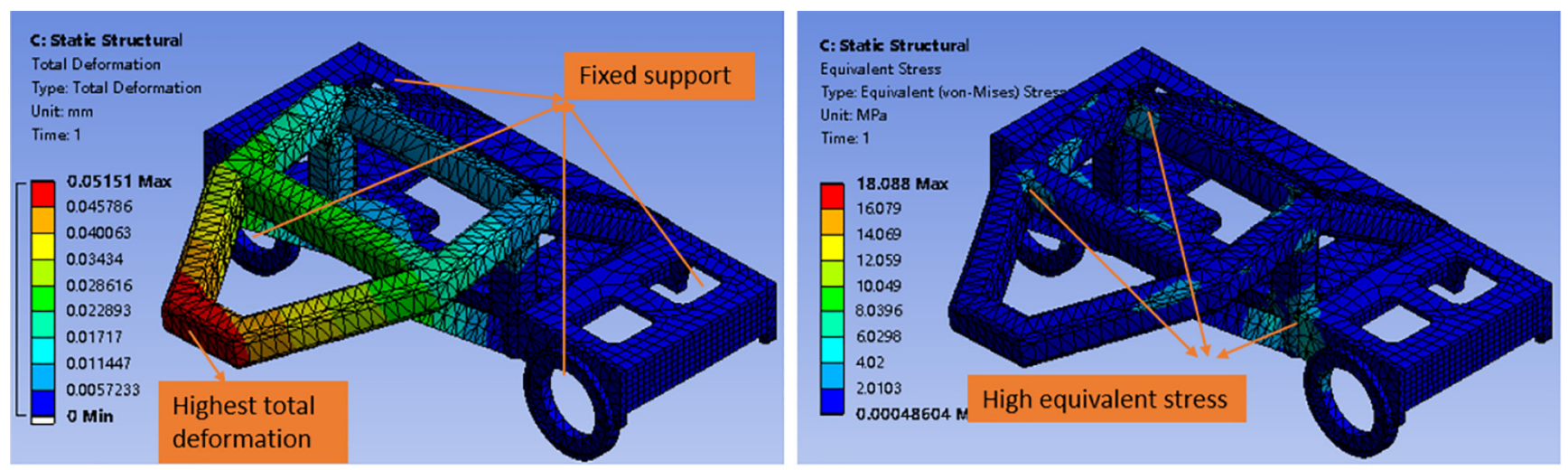

Notes: (1) Total deformation (left); (2) equivalent stresses (right) 
Figure 11 Control system hardware

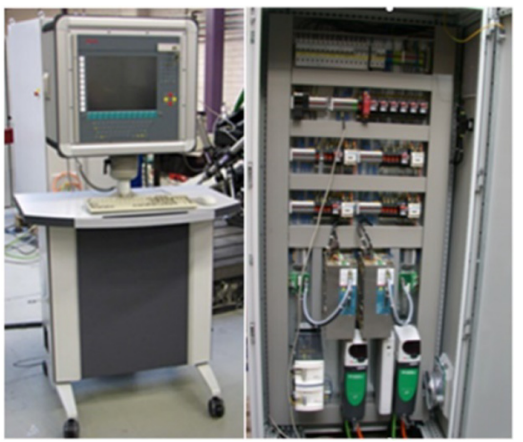

controlled in one interface. The on-site debugging and the tungsten inert gas (TIG) welding test can be seen in Figure 13 (left). The test was to weld two pieces of stainless steel of $10 \mathrm{~mm}$, and the related results shown in Figure 13 (right) reached the requirements.

\subsection{Machining experiment}

The milling process and its result can be seen in Figure 14. Several materials were tested, such as plastic and aluminum alloy. The milling spindle in this robot combines the spindle motor and the machine tool spindle mounted on a multifunction flange. It can carry different tasks, such as machining, deburring, drilling and polishing. In our work, the milling task was done with small feed in specific location of the VV; the

Figure 12 Structure of control system, digital twins and real robot machine

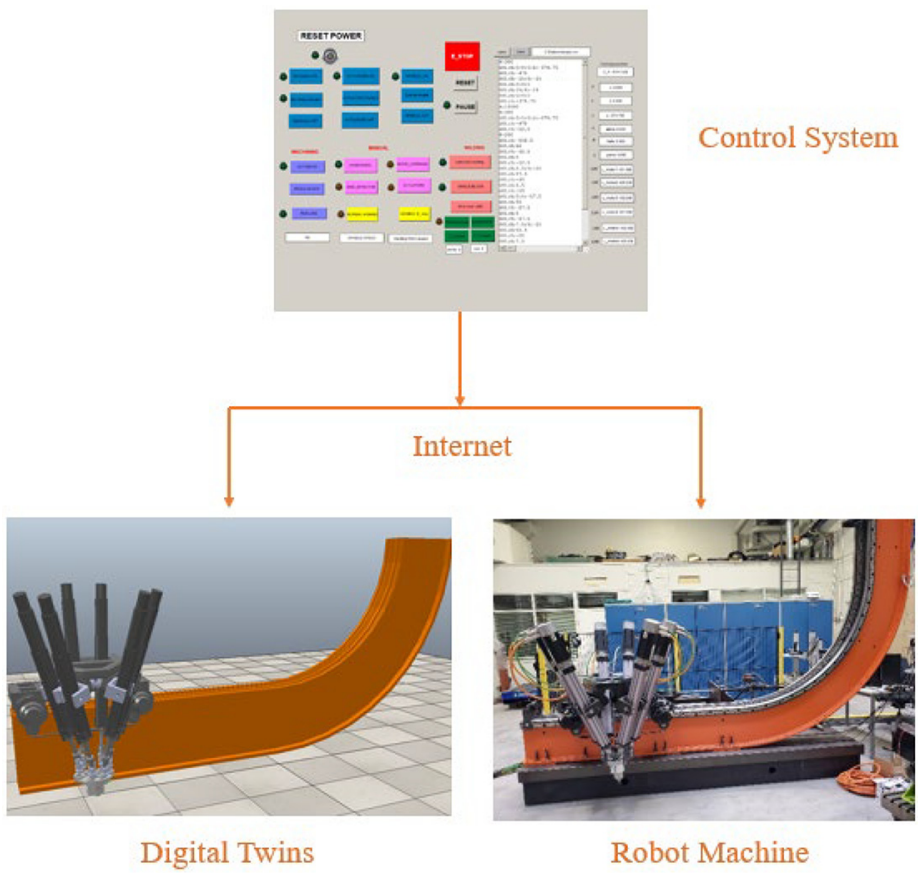

the track rail from horizontal position to vertical position with 0.1-mm movement accuracy. As for the parallel manipulator, the movable platform can reach any position in the box of $200 \times 200 \times 300 \mathrm{~mm}(x-y-z)$.

\subsection{Welding experiment}

The ESAB welding software is integrated with the robot control software, and the feeding system and welding system can be

Figure 13 Welding test: welding process (left) and welding result (right)

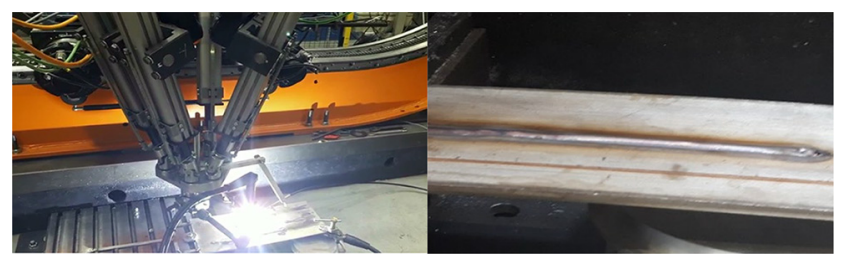

Figure 14 Milling test: milling test set up (left) and milling test accuracy check (right)
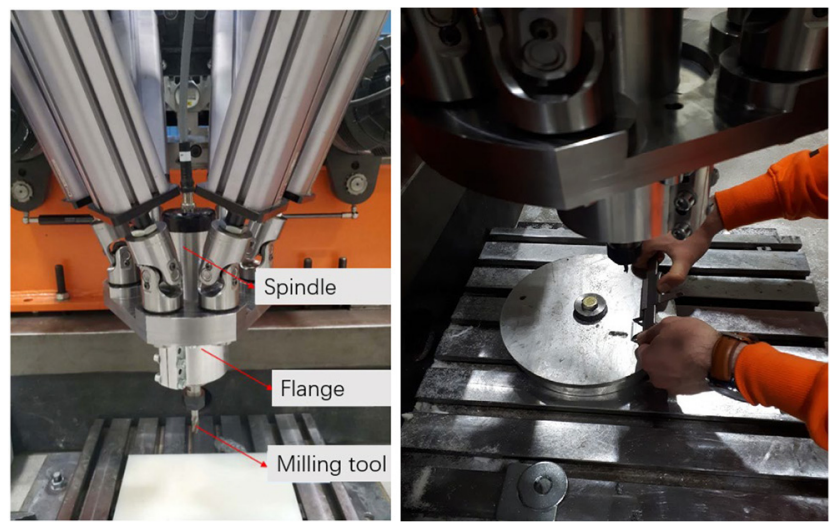
maximum rated power selected for the spindle motor was $2 \mathrm{~kW}$. During the milling process, the robot was stable with 0.1 mm milling accuracy.

\section{Conclusion}

A mobile parallel robot with seven degrees of freedom driven by the electrical motor and actuators was designed and manufactured for the assembling tasks of fusion reactor. The control system was debugged and tested, and the welding and machining processes were implemented in the laboratory. This robot can carry out tasks with the required accuracy. The kinematics, dynamics and the finite element analysis of this mobile parallel robot were introduced. The compact size of robot is suitable for the maintaining and assembling the fusion reactors of different sizes, such as the DEMO and the CFETR. Our future work is to focus on the multi-objective design of parallel robot by means of the kinematics model, the dynamic model and the workspace volume, while the design variables will be the geometry of the parallel robot, such as the diameters of the platforms and the location of the actuators. The goal is to develop an algorithm as a tool to design the parallel manipulator in a systematic and reliable way.

\section{References}

Feng, Y., Yu, X. and Man, Z. (2002), "Non-singular terminal sliding mode control of rigid manipulators", Automatica, Vol. 38 No. 12, pp. 2159-2167, doi: 10.1016/S0005-1098(02)00147-4.

Li, C., Wu, H., Eskelinen, H., Siuko, M. and Loving, A. (2019), "Design and analysis of robot for the maintenance of divertor in DEMO fusion reactor", Fusion Engineering and Design, Vol. 146, pp. 2092-2095, doi: 10.1016/j. fusengdes.2019.03.110. (September 2018)

Li, C., Wu, H., Eskelinen, H., Handroos, H. and Li, M. (2020), "Design and implementation of a mobile parallel robot for assembling and machining the fusion reactor vacuum vessel", Fusion Engineering and Design, Vol. 161, p. 111966, doi: 10.1016/j.fusengdes.2020.111966. (September 2019)
Li, M., Wu, H., Handroos, H. and Yang, G. (2013), "Software design of the hybrid robot machine for ITER vacuum vessel assembly and maintenance", Fusion Engineering and Design, Vol. 88 Nos 9/10, pp. 1872-1876, doi: 10.1016/j. fusengdes.2013.04.023.

Merlet, J.P. (2006), "Parallel robots", Solid Mechanics and Its Applications, doi: 10.1007/978-3-319-72911-4_6.

Pessi, P., Wu, H., Handroos, H. and Jones, L. (2007), “A mobile robot with parallel kinematics to meet the requirements for assembling and machining the ITER vacuum vessel", Fusion Engineering and Design, Vol. 82 Nos 15/24, pp. 2047-2054, doi: 10.1016/j. fusengdes.2007.06.012.

Song, Y.T., Wu, S.T., Li, J.G., Wan, B.N., Wan, Y.X., Fu, P., Ye, M.Y., Zheng, J.X., Lu, K., Gao, X., Liu, S.M., Liu, X. F., Lei, M.Z., Peng, X.B. and Chen, Y. (2014), "Concept design of CFETR tokamak machine", IEEE Transactions on Plasma Science, Vol. 42 No. 3, pp. 503-509, doi: 10.1109/ TPS.2014.2299277.

Stewart, D. (1965), "A platform with six degrees of freedom", Proceedings of the Institution of Mechanical Engineers, Vol. 180 No. 1, pp. 371-386, doi: 10.1243/ pime_proc_1965_180_029_02.

Ting, Y., Chen, Y.S. and Jar, H.C. (2004), "Modeling and control for a Gough-Stewart platform CNC machine", fournal of Robotic Systems, Vol. 21 No. 11, pp. 609-623, doi: 10.1002/rob.20039.

Wang, Z., Wang, Z., Liu, W. and Lei, Y. (2001), "A study on workspace, boundary workspace analysis and workpiece positioning for parallel machine tools", Mechanism and Machine Theory, Vol. 36 No. 5, pp. 605-622, doi: 10.1016/S0094-114X (01)00009-X.

Wu, H., Handroos, H. and Pessi, P. (2008), "Mobile parallel robot for assembly and repair of ITER vacuum vessel", Industrial Robot: An International fournal, Vol. 35 No. 2, pp. 160-168, doi: 10.1108/01439910810854656.

\section{Corresponding author}

Changyang Li can be contacted at: changyang.li@lut.fi 